\title{
The Theory and Technique of Yamuna Body Rolling
}

\author{
SAtoshi SuZuki, ATC, $\operatorname{CSCS}^{1)}$ \\ 1) Department of Rehabilitation, Jiyugaoka Orthopedic Clinic: 1-15-12 Tamagawa Denenchofu, \\ Setagaya-ku, Tokyo 158-0085, Japan. TEL +81 3-3772-1411, FAX: + 81 3-3772-1411
}

\begin{abstract}
Purpose] This paper provides information about the theory and technique of Yamuna Body Rolling. In order to treat physical problems, using the specialized Yamuna Body Rolling balls, people can target superficial skin, fasciae, muscle fibers, tendons, ligaments, bones, internal organs, and the nervous system by themselves. The extraordinary effect of Yamuna Body Rolling is its multidimensional elongation of muscle fibers. In addition to the regular longitudinal elongation by the conventional stretch method, Yamuna Body Rolling enables the transversal and diagonal expansion of muscle fibers in order to move the body more dynamically. Hamstring, abdominal, and sideline routines are presented as examples for techniques of Yamuna Body Rolling. Yamuna Body Rolling can be applied to functional evaluation and therapeutic uses; therefore, it could provide many benefits in the treatment of different conditions in the medical field.

Key words: Yamuna, Muscle elongation, Self-conditioning
\end{abstract}

(This article was submitted Feb. 27, 2013, and was accepted Apr. 19, 2013)

\section{INTRODUCTION}

Recently, in order to relieve daily aches and improve quality of life, many kinds of self-conditioning tools have gained in popularity. Yamuna Body Rolling (YBR; the name commonly used), which was originally developed in New York, the United States of America, is one of the selfconditioning methods using small specialized balls. The demand for YBR has been increasing all over the world. In order to treat physical problems, using the YBR balls, people can target superficial skin, fasciae, muscle fibers, tendons, ligaments, bones, internal organs, and the nervous system by themselves. The unique method has aspects of both exercise and treatment. Leading individual body parts to their accurate positions, YBR makes necessary space in the body, increases circulation, and improves range of motions.

The purpose of this paper is to show health-care providers that this method can be extremely useful in treating and curing musculoskeletal problems. YBR can also be used clinically to improve internal organs and the circulatory and nervous system functions. Future studies to show how direct external stimulation applied anatomically correctly to bones and muscles can be used not only to improve the musculoskeletal system but also the internal systems is highly suggested.

${ }^{*}$ To whom correspondence should be addressed. E-mail: suzukisatoshi@hotmail.com

\section{THEORY}

\section{What is Yamuna?}

Yamuna Zake created the total bodywork system generally named Yamuna after her in New York in the United States in 19791). The term of Yamuna is a registered trademark. Yamuna mainly consists of four different categories: "Yamuna Body Logic," "Yamuna Body Rolling," "Yamuna Foot Fitness," and "Yamuna Face Ball." Zake created the manual therapy called Yamuna Body Logic first. YBR was developed from Yamuna Body Logic to maintain the conditions of therapists and provide a home program for patients. YBR has the same therapeutic benefits as Yamuna Body Logic and uses a specialized exercise ball as a self-conditioning tool.

This unique program gives people distinct methods to work on themselves in order to sustain their postures, flexibilities, strengths, and alignments throughout their lives. Yamuna is a totally different concept compared with conventional "fitness" regimes and newly categorized as "Body Sustainability." It has been widely used in many countries in North America, Europe, South America, and Oceania and in Japan.

Yamuna is based theoretically on people performing certain movements on a specialized ball called "routines" by controlling their own body weight and stimulating muscular origins, tendons, muscle bellies, and insertions. Repeating deep inhalation and exhalation stimulates the parasympathetic nervous system, relaxing muscle tensions and making the body weight sink into the ball. After sinking into the ball as the muscles elongate, the person distally stretches or, in other words, self-tractions the intended body parts to 


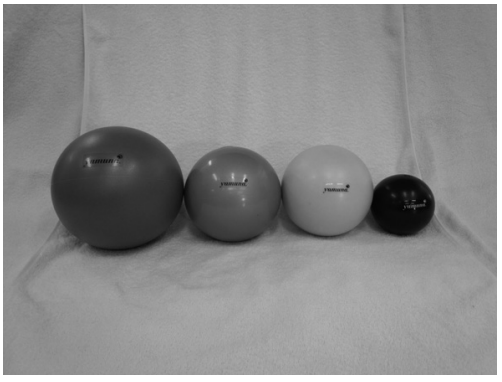

Fig. 1. Yamuna Balls From left to right: gold ball, silver ball, pearl ball, and black ball.

increase the joint play. During stretching out of limbs, any rotational malalignment can also be adjusted. After one side is done, it is very important to evaluate and compare the conditions of the worked side to the other side. It is not recommended to forcefully press toward the ball because this activates the stretch reflex as an opposing effect.

The extraordinary effect of YBR is its multidimensional elongation of muscle fibers. In addition to the regular longitudinal elasticity resulting from the conventional stretch method, the transversal and diagonal expansion of muscle fibers by YBR enables the body to move more dynamically. Also, YBR can easily access to abdominal muscles and internal organs even though regular stretching cannot usually target those sensitive areas. For example, YBR can expand the rib cage to increase the lung capacity.

\section{YBR Ball}

The YBR ball is specially made to fit uneven surfaces of the musculoskeletal system with its appropriate hardness and flexibility. The material is customized polyvinyl chloride, and the safety with regards to the human body is guaranteed by Cosmetics Directive 76/768/EEC of the European Commission and Directive 2005/84/EC of the European Parliament. All five YBR balls hold up to $160 \mathrm{~kg}$.

The biggest ball, the gold ball, is $25 \mathrm{~cm}$ in diameter and is usually used by beginners and for sensitive areas such as the rib cage and abdomen since the pressure toward the body it produces is the lowest. Slightly smaller than the gold ball, the pearl ball is $15 \mathrm{~cm}$ in diameter and made for a relatively smaller person and travel usage.

The denser silver ball is $23 \mathrm{~cm}$ in diameter and is used for stimulating especially lower extremities by advance users. For small body parts such as the calf and hip, the calf (black) ball, which is $10 \mathrm{~cm}$ in diameter, is suitable. Recently, a blue ball made with the same hardness as the silver ball has been produced for advanced usage as a calf ball. The face ball is customized for facial use, and hemisphereshaped foot wakers and foot savers can especially stimulate the feet. By choosing and using a variety of tools, total body self-conditioning is possible (Fig. 1).

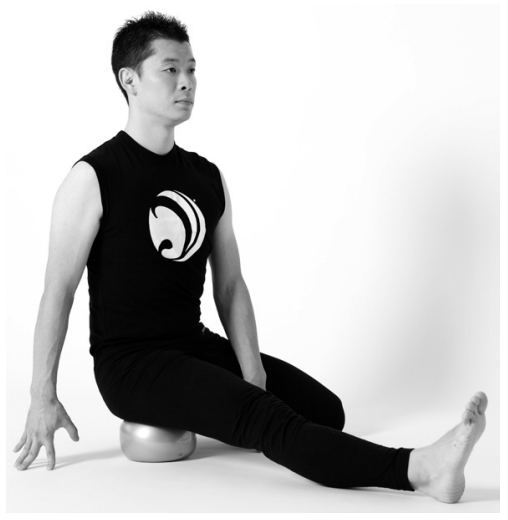

Fig. 2. Hamstrings Routine

\section{TECHNIQUE}

Hamstring, abdominal, and sideline routines are presented in this chapter as starting on the right side.

\section{Hamstrings Routine ${ }^{2)}$}

Sit with the ball under the right ischium and the right leg proximally extending out as straight as possible. The rotation of the hip joint is in a neutral position. Tilt the pelvis anteriorly to straighten the upper body upward. Bend the left leg with the foot flat on the floor for stability and move the body. Also, press the fingertips against the floor for balance.

Bear weight downward toward the ischium as you roll on the ball forward and backward, side to side, clockwise and counterclockwise to sterically stimulate the origins of the semimembranosus, semitendinosus, long head of the biceps femoris, and adductor magnus. Then, staying on the center of the ischium, breathe deeply two to three times, relax and, sink the body weight toward the ball as you exhale.

After stimulating the ischium, move the body backward about $1 \mathrm{~cm}$, and place the ball under hamstring tendons. Move the body side to side to distinguish the tendon fibers. Sink the body into the ball and then stretch the leg out after feeling the muscles relax. Next, move the body to stimulate the muscle belly.

Repeat breathing deeply, sinking in, and stretching outward at each point of the muscle as you move the ball in $5-\mathrm{cm}$ increments until it reaches the distal $3 / 4$ of the posterior femur, and then roll the ball back to the ischium. Slowly get off the ball, sit on the floor with both legs extended, and observe the difference between the two sides. Stand and walk to feel the conditions of the legs also. Repeat the above sequences on the left side.

During the entire routine, it is important to maintain the anterior tilt of the pelvis and distally extend the right leg, increasing the hip joint play by separating the femur out from the acetabulum. With this multidimensional elongation of the muscles, rotational misalignment of the hip can be adjusted. In addition to the basic routine, other variations can focus on each of the individual hamstring muscles, and an advanced routine can separate the muscles transversally. Stimulation of the popliteal fossa for an inflamed or painful knee is contraindicated (Fig. 2). 


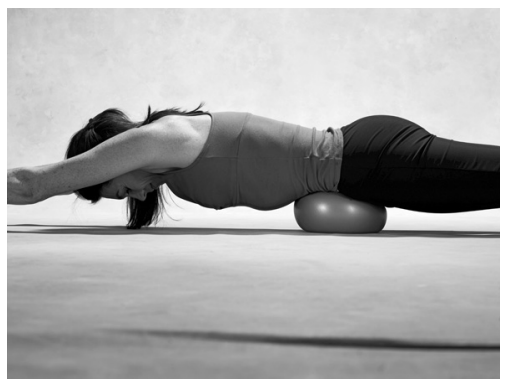

Fig. 3. Abdominal Routine

\section{Abdominal Routine $e^{2}$}

In the prone position, place the ball at the right anterior posterior iliac spine with the leg extended straight outward, and with 90 degrees flexion of both shoulders and elbows, put the forearms on the floor to stabilize the body. The toes of the right foot should be on the floor with the foot flexed. The right knee should be off the floor, but the left knee should rest on the floor. Keep the left pelvis at the same height as the right side to optimally bear weight on the ball. It is also important to maintain a neutral position of the scapulas and lumbar spine in order to not put extra stress on the body.

After stimulating the right anterior posterior iliac spine with two to three deep breaths, slowly roll the ball off the bone into the abdomen along the curve of the ilium. The right arm should be extended over the head, and with the dorsal foot resting on the floor, the right leg should be distally stretched to separate the trunk and leg. Repeat deep inhalation and exhalation to sink the body into the ball.

The right pelvis tilts posteriorly and sinks into the ball toward the floor, stretching not only the abdominal area but also the lower back at the same time. Repeat sinking into the ball and extending the arm and leg two to three times, and then slowly come off the ball as you roll the body in a supine position. Feel the difference on the other side, and repeat on the left side.

This routine affects not only the musculoskeletal system such as the sartorius, tensor fasciae latae, iliacus, psoas major, internal oblique, external oblique, transverse abdominis and hip, sacroiliac, and lumbosacral joints but also internal organs such as the large and small intestines, urinary system, and reproductive system. Traditional stretching does not provide as much benefits.

Another variation starts with stimulation of the pubic symphysis, and the ball is then rolled upward along the center line of the abdominal area. Any surgery in the abdominal area within 12 weeks is contraindicated. To prevent potential damage, do not directly stimulate any floating ribs (Fig. 3).

\section{Sideline Routine ${ }^{2)}$}

Place the ball under the right greater trochanter and stabilize the body as straight as possible with the right hand or forearm on the floor. Breathe deeply to sink the body into the ball. Next, move the body downward to stimulate the iliac crest, and lift up the right rib cage with the left hand to

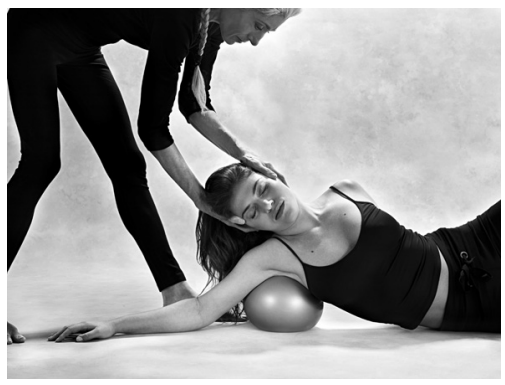

Fig. 4. Sideline Routine separate the rib from the pelvis. After a few breaths, slowly roll off the ball.

Lay on your right side and place the ball under the area near the right seventh rib. Extend and straighten the right leg out, and place the right forearm and left hand on the floor to stabilize the body. Bend the left knee with the foot flat on the floor for additional support. Take an exaggerated deep breath to expand the right rib cage laterally toward the ball, keep extending the right leg distally, and lift the whole upper body upward to separate from the pelvis.

Move the body downward to stimulate around the fifth rib. Bend the right elbow and place it on the floor; then rest the head firmly on the right hand. Repeat deep breathing a few times to widen the rib cage. Next, place the ball under the armpit, and raise the arm overhead as long as possible. Place the left fist in between the head and right side of the neck to stabilize the head. Spread the pectoralis major and latissimus dorsi to make room for the ball to firmly stimulate the second or third rib, the humerus head, and the lateral border of the scapula. The purpose is to separate the scapula from the thorax and the humerus from the glenoid cavity.

Afterward, move the body downward to place the ball under the proximal humerus, and repeat breathing to extend the arm further. Now, go back to axillary fossa to relax the shoulder again and come off the ball as you roll in a supine position. Evaluate the expansion of the right thorax and the range of motion as you raise the arm overhead as far upward as possible (Fig. 4).

\section{CLINICAL APPLICATION}

YBR provides a variety of self-treatment programs for use at home or anywhere. It also reduces the risk of potential harm to muscles, although static stretching might strain fasciae and muscle fibers with excessive elongation force. While static stretching utilizes the joint motions to indirectly elongate muscle in only the longitudinal direction, some of the intended muscle fibers are possibly unaffected. Moreover, longitudinal expansion possibly compresses capillary vessels and reduces circulation.

However, YBR is the most complete form of stretching because it can directly and evenly stimulate tendons and muscle fibers, increasing movement through the entire muscle. Also, due to stimulation of Golgi tendon organs at myotendinous junction even relaxes the affected muscles 
known as autogenic inhibition for further stretching effect. Even though it is hard for traditional stretching to target certain body parts such as the abdomen, thorax, adductors, hip rotators, and spine, these areas can be adequately elongated using the specialized ball. In addition, YBR produces mechanical stress on the anterior, posterior, medial, and lateral surfaces of bones, possibly facilitating the remodeling process; most weight bearing physical activities load bone only in the axial direction.

Moreover, YBR safely maintains or increases the range of motion of an injured joint. Taking an ankle sprain as an example, you sit on the floor with the leg extended and place a ball under the knee to start stimulating the calf muscle. You then move the ball toward the ankle in small increments and use your hands or other leg to gently press the calf muscle into the ball. Without any forceful dorsi flexion of the inflamed ankle joint, the calf muscle can be stretched to prevent minimizing its range of motion.

The safety of static stretching is dependent on the skill levels of therapists and stretchers. Loading excessive force on muscles stimulates the stretch reflex, which inversely affects the intended muscles, causing the person to not receive an appropriate result. Furthermore, overstretching even causes micro tearing of muscle fibers. However, the method of YBR is relatively harmless to people because it utilizes deep breathing to relax the muscles without applying any vigorous pressure.

In the case that a person cannot physically perform YBR on their own, a therapist can treat them with a ball and the YBR method as a hands-on therapy while they are lying on a table. Therapeutic usage of YBR benefits clients in a va- riety of manners all at once, including traction, stretching, mobilization, massage, and muscle activation. In addition, the therapist can appropriately treat sensitive body parts, for instance, areas around the pelvic region without any discomfort for the client.

\section{FUTURE RESEARCH}

For clinical application with regards to specific disorders, we would like to research effective methods for different types of muscles and joints, the appropriate amount of pressure to apply to each body part and conditions, and scientific evidence for the duration of effectiveness with randomized controlled trials.

\section{CONCLUSION}

This paper introduces the principles of YBR. My personal opinion is that YBR is a very effective approach in many clinical settings because it evaluates and treats conditions simultaneously and makes clients more independent. I hope that YBR will be used as a self-conditioning method for not only healthy people but also patients who require physical therapy in clinical fields.

\section{REFERENCES}

1) Zake Y, Golden S: Body Rolling. An Experiential Approach to Complete Muscle Release. Healing Arts Press, 1997.

2) Zake Y, Golden S: The Ultimate Body Rolling Workout. The Revolutionary Way to Tone, Lengthen, and Realign Your Body. Three Rivers Press, 2003. 